This paper is a postprint of a paper submitted to and accepted for publication in IET Optoelectronics and is subject to Institution of Engineering and Technology Copyright. The copy of record is available at IET Digital Library

\title{
Performance Evaluation of Digital Pulse Position Modulation for WDM FSO Systems Impaired by Interchannel Crosstalk
}

$\dagger$ A.M. Mbah, J.G. Walker, A.J. Phillips

Division of Electrical Systems and Optics, Faculty of Engineering, University of Nottingham, University Park, Nottingham NG7 2RD, UK

$†$ Corresponding author's email: eexammb@nottingham.ac.uk

Tel: +44 (0) 115846 6708, Fax: +44 (0) 1159515616

\begin{abstract}
Wavelength division multiplexing (WDM) has been proposed for fibre, intersatellite, free space and indoor optical communication systems. Digital pulse position modulation (DPPM) is a more power efficient modulation format than on-off keying $(\mathrm{OOK})$ and a strong contender for the modulation of free space systems. While DPPM obtains this advantage in exchange for a bandwidth expansion WDM systems using it are still potentially attractive, particularly for moderate coding levels. However WDM systems are susceptible to interchannel crosstalk and modelling this in a WDM DPPM system is necessary. Models of varying complexity, based on simplifying assumptions, are presented and evaluated for the case of a single crosstalk wavelength. For a single crosstalk, results can be straightforwardly obtained by artificially imposing the computationally convenient constraint that frames (and thus slots also) align (FA). Multiple crosstalk effects are additionally investigated, for the most practically relevant
\end{abstract}


cases of modest coding level, and using both simulation and analytical methods. In general, DPPM maintains its sensitivity advantage over OOK even in the presence of crosstalk while predicting lower power penalty at low coding level in WDM systems.

\section{Introduction}

Digital pulse position modulation (DPPM) is well known to be an attractive modulation format in free space optical communications (whether intersatellite, atmospheric or indoor wireless) [1-3]. Apart from the power efficiency advantage there is the additional advantage in some schemes, like the one considered here, that there is no need to set and track a decision threshold $[4,5]$. DPPM is particularly attractive over this channel relative to a fibre channel (which has been proposed and intensively investigated $[6,7])$, because the channel is dispersion free [1]. The advantages of DPPM however do come at the expense of a bandwidth expansion.

With the continuous increase in demand for bandwidth, wavelength division multiplexing (WDM) systems have been investigated and/or deployed for fibre, free space and wireless optical networks [8-10]. Multi-user access network scenarios may also be served by WDM, for example WDM passive optical network (PON) is generally considered as a good solution to the bandwidth requirement for future access networks, with potential for higher data rate, improved data security and longer reach $[8,11]$. The drivers for WDM deployment remain the same whether the modulation format is conventional on off keying (OOK) or DPPM (as long as the bandwidth expansion can be incorporated). Although the improvement in receiver sensitivity comes at the expense of bandwidth, with a moderately low coding level, DPPM can combine with most multiplexing/multiple access schemes and other modulation techniques without considerable bandwidth expansion. For example, some variants and hybrids of DPPM with techniques such as phase shift keying (PSK) and frequency shift keying (FSK) have been 
proposed for point-to-point fibre communication systems [12, 13]. WDM systems using DPPM have been studied in [14-17]. However, once there are multiple wavelengths in a communication link, imperfect optical components (e.g. demultiplexers, filters, etc. $[18,19])$ and possibly asymmetric loss necessitate the evaluation of the impact of interchannel crosstalk $[20,21]$. This is well known for OOK; crosstalk in WDM OOK systems has been investigated for fibre, intersatellite and wireless links [18, 20, 22-24]. However, such a performance evaluation for DPPM interchannel crosstalk has not yet been provided (for any of the realistic WDM DPPM scenarios whether intersatellite, atmospheric, indoor, multi-user, PON or point to point). This paper is intended to remedy this shortfall. It should be noted that necessarily this evaluation is somewhat more complex than the equivalent for OOK.

Specifically in this paper, the evaluation of an optically preamplified WDM DPPM wireless system impaired by interchannel crosstalk is performed. The presence of an optical amplifier (OA) further improves the receiver sensitivity, but introduces amplified spontaneous emission (ASE) noise. The ASE noise beats with the signal and itself to produce signal-spontaneous and spontaneous-spontaneous beat noises which degrade the system performance. Established bit error rate (BER) evaluation techniques including the Gaussian approximation (GA), Chernoff bound (CB) and modified Chernoff bound (MCB) are applied in the analysis. While the GA uses only means and variances to describe the signal and noise, the $\mathrm{CB}$ and $\mathrm{MCB}$ use the moment generating function (MGF) which gives a full statistical description of the signal and noise [7]. Results obtained analytically (and in the case of multiple crosstalk verified with Monte Carlo simulations) are compared with WDM OOK results and presented. However experimental verification would be necessary for full system characterization. A foreseeable issue in experimentation is the complexity involved in symbol synchronization, and a possible solution may include the method employed in [25]. 


\section{Optically Preamplified WDM DPPM receiver}

In DPPM signal transmission format, a frame of duration equal to $M T_{b}$ is divided into $n=2^{M}$ equal time slots of length $t_{s}=M T_{b} / n$, where $M$ is the coding level and equal to the number of data bits transmitted per DPPM frame and $T_{b}=1 / R_{b}$ is the equivalent OOK NRZ bit period, where $R_{b}$ is the bit rate. The maximum likelihood detection receiver is preferred for the best performance in DPPM optical wireless systems [1]. Circuitry is required to integrate over each slot in a frame and the decision is made by comparing the results and selecting the slot with the largest signal as the pulse position [4].

A general WDM DPPM system that might require evaluation of crosstalk impact could include a fibre or free space (or hybrid) channels and may be in a point-to-point, multipoint-to-point or PON configuration. Different sources and levels of crosstalk could arise in a WDM DPPM system depending on the link configuration. In most point-to-point systems with all signal wavelengths originating from the same place, the major source of crosstalk is imperfect optical bandpass filter (OBPF)/demultiplexer (demux) rejection and since most realistic systems will employ OBPF/demux with good rejection ratio, unless there is a power drop in the signal wavelength compared to the interfering wavelengths (or some relative spectral shift of passband and signal), the crosstalk level will be fairly small. This is also the case in point-tomultipoint fibre systems (like WDM PON downstream) with all signal wavelengths originating from the same place. But in multipoint-to-point links such as upstream transmission in hybrid fibre and FSO systems or in PON (where signals could experience asymmetric splitting loss, fibre and/or FSO attenuation, beam spreading and coupling loss), signals at different wavelengths will arrive at the $\mathrm{OBPF} / \mathrm{demux}$ at different power levels. Under this condition, the crosstalk in the system is no longer dependent only on the OBPF/demux channel rejection but could rise much higher depending on the difference between the signal power of the interfering 
wavelengths and the desired wavelength at the input of the optical filter. Other cases where asymmetry could affect the level of crosstalk include point-to-multipoint systems with signal wavelengths having different powers prior to multiplexing.

A generic system structure which could be easily adapted to all the different scenarios above is shown in Fig. 1. DPPM signals from different wavelengths are multiplexed and transmitted over a wireless/free-space link to a receiving lens. They could also in principle arise from different physical locations as long as they can be collected and coupled effectively into the optical amplifier (OA) which is done by collimating them into a short fibre length at the amplifier input before being demultiplexed into different wavelengths for detection by a PIN photodiode. The optical preamplifier is just treated as a linear gain block generating noise as in Fig. 1. Thus saturation based effects, and other nonlinearities, that may justify a more sophisticated treatment to include the contribution of certain optical amplifiers to the overall crosstalk at the receiver, are not incorporated. The demux/OBPF provides an effective optical bandpass filtering which helps to reduce the ASE noise prior to photodetection, and the detected signal is passed through electrical filtering and amplification before integrate and compare circuitry is used to decide which DPPM slot contains the signal pulse. Finally, the $M$ bit word corresponding to the chosen slot is selected as the receiver output.

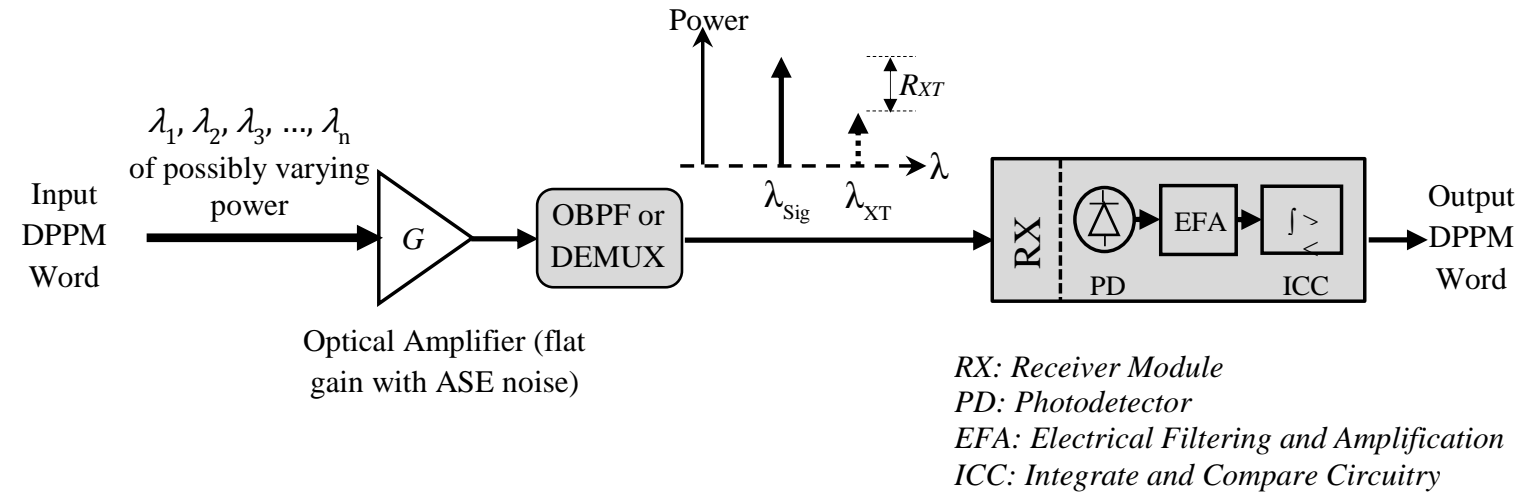

Fig. 1: Generic system structure for optically preamplified WDM DPPM receiver 


\section{Crosstalk Modelling}

The analysis of crosstalk in a DPPM system requires some consideration to ensure that the different scenarios that could arise during frame reception are taken into account. For example, there may be assumed (and it is stressed that this is generally for mathematical convenience) an alignment of frames (and evidently slots) (FA) or only slots (OSA) between the signal and crosstalk (XT) as shown in Figs. 2a and 2 b respectively. However, in a practical system, it is more likely that there is a misalignment of slots (SM) (and evidently frames) between signal and crosstalk during signal reception (see Fig. 2c). In Fig. 2, $\left\{n_{1}, n_{2}\right\} \in \mathbb{Z}$ (integer) are the number of whole slots in the earlier and later transmitted crosstalk frames respectively that overlap the signal frame under consideration, while $t_{1}\left(\right.$ or $\left.t_{2}\right)$ is the slot offset between the slots in a particular signal frame and the slots in the earlier (or later) transmitted crosstalk frame that overlap with the signal frame, also $t_{2}=t_{s}-t_{1}$. Thus both $t_{1}$ and $t_{2}$ define the fractional or partial crosstalk that could affect the signal slots. Furthermore, in the case of both OSA and SM there is the possibility in some systems that the misalignment is maintained for a long time period and thus performance would be calculated for the specific misalignment. Equally in many realistic systems the misalignment will change sufficiently frequently that the proper evaluation approach is to average over all different (mis)alignments. 

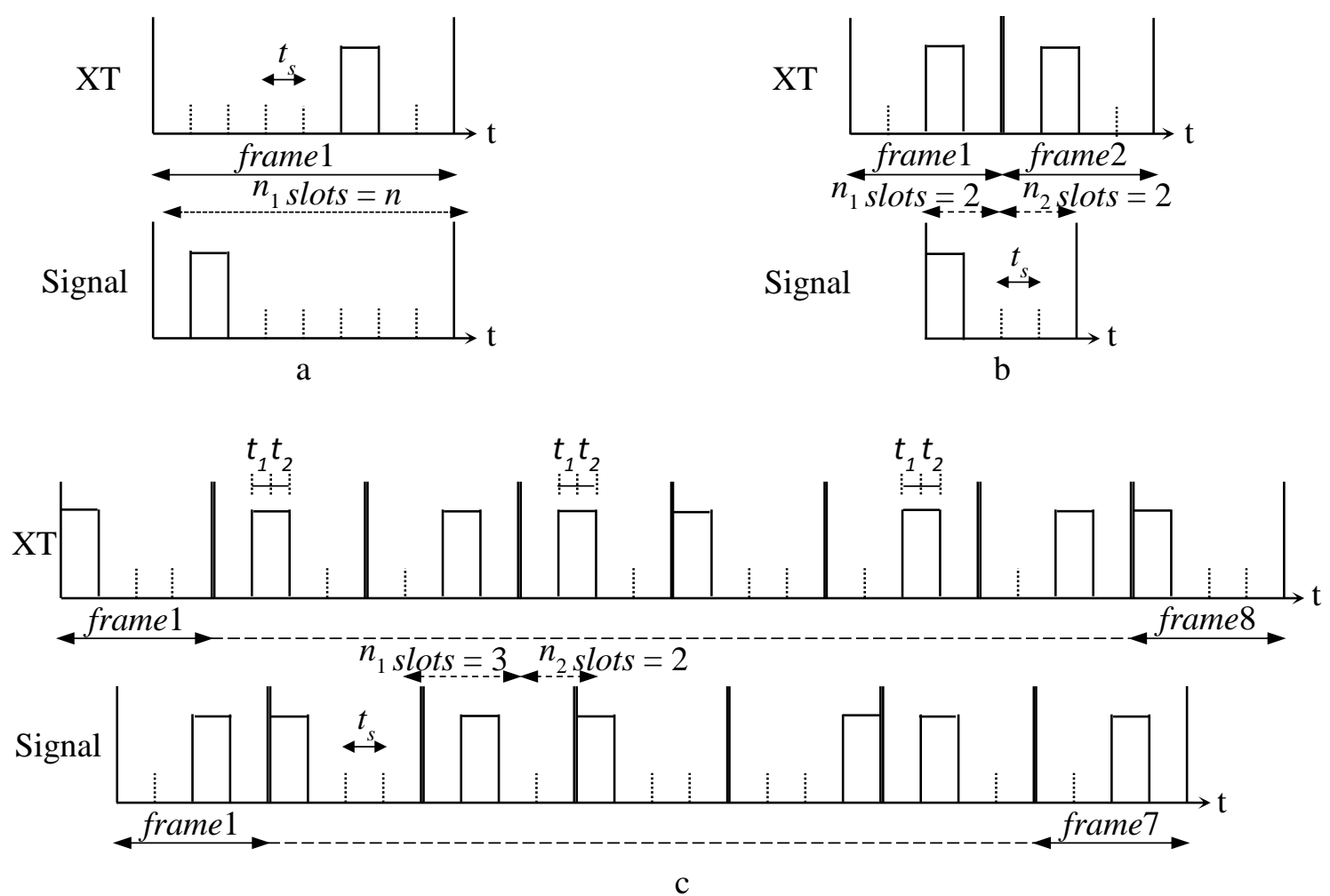

Fig. 2: Illustration of crosstalk in WDM DPPM receiver (a) Frames aligned (FA) for $M=3$, (b)

Only slots aligned (OSA) for $M=2$ and (c) Slots (and frames) misaligned (SM) for $M=2$ 
Table 1: List of Probability Parameters

\begin{tabular}{|c|c|}
\hline Notation & Description \\
\hline$j, j_{1}$ & $\begin{array}{l}\text { Number of crosstalk pulses (from crosstalk sources with frames misaligned } \\
\text { with signal frame) that occur in signal slot }(j) \text { and empty slot }\left(j_{1}\right) \text { of the } \\
\text { signal. }\end{array}$ \\
\hline$k, k_{1}$ & $\begin{array}{l}\text { Number of crosstalk pulses (from crosstalk sources with frames aligned } \\
\text { with the signal frame) that occur in the signal slot }(k) \text { and empty slot }\left(k_{1}\right) \\
\text { of the signal frame. }\end{array}$ \\
\hline$l_{i}$ & $\begin{array}{l}\text { Number of full }\left(l_{s}\right) \text { or partial }\left(l_{1}, l_{2}\right) \text { crosstalk pulses occurring in the entire } \\
\text { signal frame. }\end{array}$ \\
\hline$n_{1}, n_{2}$ & $\begin{array}{l}\text { Number of full slots in the earlier }\left(n_{1}\right) \text { and later }\left(n_{2}\right) \text { transmitted crosstalk } \\
\text { frames that overlap with the signal frame under consideration. }\end{array}$ \\
\hline$p_{f\left(l_{i}\right)}\left(n_{1}\right)$ & $\begin{array}{l}\text { Probability of } l_{i}\left(l_{s}, l_{1} \text { and/or } l_{2}\right) \text { crosstalk occurring in the signal frame } \\
\text { when } n_{1} \text { slots overlap that particular signal frame. }\end{array}$ \\
\hline$p_{s\left(l_{i}\right)}\left(r_{i}\right)$ & $\begin{array}{l}\text { Probability of } r_{i}\left(r_{s}, r_{1} \text { and/or } r_{2}\right) \text { crosstalk occurring in the signal pulse } \\
\text { slot when } l_{i}\left(l_{s}, l_{1} \text { and/or } l_{2}\right) \text { crosstalk occur in the signal frame. }\end{array}$ \\
\hline$P_{\text {we }\left(l_{i}-r_{i}\right)}$ & $\begin{array}{l}\text { Symbol error probability with } l_{i}\left(l_{s}, l_{1} \text { and/or } l_{2}\right) \text { crosstalk in the signal } \\
\text { frame and } r_{i}\left(r_{s}, r_{1} \text { and/or } r_{2}\right) \text { crosstalk in the signal pulse slot. }\end{array}$ \\
\hline$r_{i}$ & $\begin{array}{l}\text { Number of full }\left(r_{s}\right) \text { or partial }\left(r_{1}, r_{2}\right) \text { crosstalk pulse(s) occurring in the } \\
\text { signal pulse slot. }\end{array}$ \\
\hline$t_{1}, t_{2}$ & $\begin{array}{l}\text { Offset between the slots in a particular signal frame and the slots in the } \\
\text { earlier }\left(t_{1}\right) \text { and later }\left(t_{2}\right) \text { transmitted crosstalk frame that overlaps with that } \\
\text { signal frame. }\end{array}$ \\
\hline$\Delta t$ & Duration of crosstalk pulse overlap with a general slot. \\
\hline$\Delta t_{j}$ & Duration of the crosstalk overlap with the $j^{\text {th }}$ empty slot. \\
\hline
\end{tabular}

The moment generating function (MGF) describing the random variable of the current $Y_{s i g}(\Delta t)$ (where ${ }_{\text {sig }}=0$ or 1 depending on pulse transmitted or not , $\Delta t$ is the duration of the crosstalk pulse overlap with the slot under consideration) for a general slot which contains ASE, possibly 
a signal pulse and possibly a single XT pulse (or some fraction of one) is derived using the same treatment as $[21,26,27]$. It is written as:

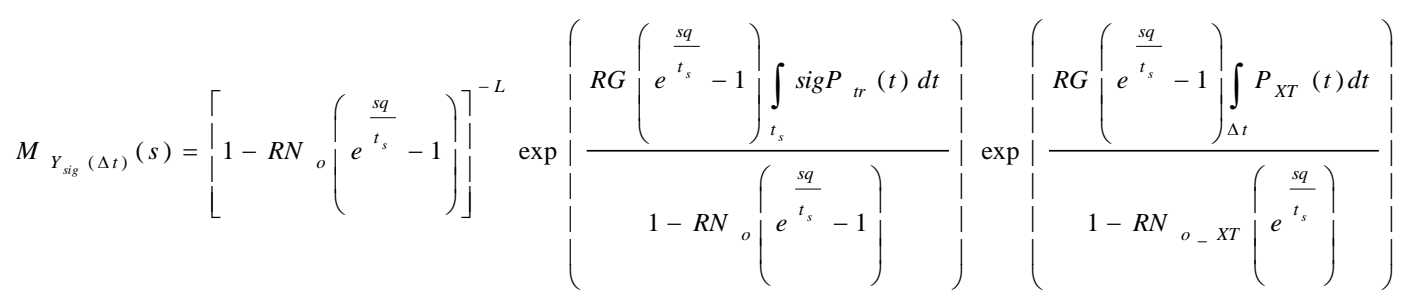

where $\Delta t=t_{s}$ if XT slots align with signal slots otherwise $t_{1}$ or $t_{2}$, and for no crosstalk in the slot, $\Delta t=0$. Also, $P_{t r}$ and $P_{X T}$ are the DPPM rectangular pulse and the crosstalk pulse power respectively, both defined at the photodetector input, $R=\eta / h v, \eta$ is the photodetector quantum efficiency, $h$ is Planck's constant, $v$ is the optical frequency, $q$ is the electron charge, $N_{o}=0.5(N F G-1) h_{v}$ is the single polarisation ASE power spectral density (PSD) at the amplifier output (and also at the photodetector input if demultiplexer nominal loss is neglected), $G$ and $N F$ are the optical amplifier gain and noise figure respectively, $L=B_{\text {opt }} m_{t} t_{s}$ is the product of spatial and temporal modes [2], $B_{\text {opt }}$ is the demux channel optical noise bandwidth and $m_{t}$ is the number of ASE noise polarisation states. $N_{o_{-} x T}$ is the ASE PSD at the photodetector at crosstalk wavelength and $R_{X T}=P_{t r} / P_{X T}$ is the signal-to-crosstalk ratio, fixed at the output of the demux. The MGF has been modified to account for crosstalk -ASE beat noise assuming the crosstalk and the desired signal experiences the same ASE noise at the amplifier output [20]. The overall MGF including the zero mean Gaussian thermal noise is given as:

$$
M_{X_{s i g}(\Delta t)}(s)=M_{Y_{s i g}(\Delta t)}(s) \exp \left(\frac{s^{2} \sigma_{t h}^{2}}{2}\right)
$$

where $\sigma_{t h}^{2}$ is the DPPM thermal noise variance. 
Following $[1,2]$, the means and variances of the random variables representing the integration over the slot that contains only the signal pulse, only crosstalk pulse, both signal and crosstalk pulses and no pulses (i.e. empty slot) are derived from the overall MGF, and are respectively generally written as:

$$
\begin{aligned}
& \mu_{X_{s i}(\Delta t)}=\frac{L R q N_{o}}{t_{s}}+R G q\left(\operatorname{sig} P_{r}+\frac{P_{X T} \Delta t}{t_{s}}\right) \\
& \sigma_{X_{s i}(\Delta t)}^{2}=\sigma_{t h}^{2}+\left(\frac{L R q^{2} N_{o}\left(1+R N_{o}\right)}{t_{s}^{2}}\right)+R G q^{2}\left[\left(1+2 R N_{o}\right) \frac{s i g P_{t r}}{t_{s}}+\left(1+2 R N_{o_{-}} X T\right) \frac{P_{X T} \Delta t}{t_{s}^{2}}\right]
\end{aligned}
$$

Given that each symbol has equal probability of being transmitted in a slot, the probability that a symbol is successfully received in the presence of crosstalk $P_{\text {ws }\left(l_{i}-r_{i}\right)}=1-P_{\text {we }\left(l_{i}-r_{i}\right)}$ where $P_{\text {we }\left(l_{i}-r_{i}\right)}$ is the symbol error probability in the presence of crosstalk, $r_{i}$ and $l_{i} \quad(i \in\{s, 1,2\})$ denote the number of crosstalk (of duration $t_{s}, t_{1}$ or $t_{2}$ ) occurring in the signal pulse slot and signal frame respectively. Thus for single crosstalk case, $r_{i} \in\{0,1\}$ while $l_{i} \in\{0,1,2\}$. Following the same treatment as [1], one can write that:

$$
\left.P_{w s\left(l_{i}-r_{i}\right)} \geq \prod_{\substack{j=1 \\ j \neq \text { sig slot }}}^{n} P\left(X_{1}(\Delta t)>X_{j}\right)\right\}
$$

where $x_{j}$ represents the content of the non-signal slot $x_{0}\left(\Delta t_{j}\right)$ and $\Delta t_{j}$ is the crosstalk overlap with the $j^{\text {th }}$ (empty) slot.

Assuming that the random variables $x_{1}(\Delta t)$ and $x_{0}\left(\Delta t_{j}\right)$ are Gaussian, the expression $P\left(X_{0}\left(\Delta t_{j}\right)>X_{1}(\Delta t)\right)$ using the Gaussian approximation (GA) of the ASE beat noises, is of the general form $[1,2]$ 


$$
P\left(X_{0}\left(\Delta t_{j}\right)>X_{1}(\Delta t)\right)=0.5 \operatorname{erfc}\left(\frac{\mu_{X_{1}(\Delta t)}-\mu_{X_{0}\left(\Delta t_{j}\right)}}{\sqrt{2\left(\sigma_{X_{1}(\Delta t)}^{2}+\sigma_{X_{0}\left(\Delta t_{j}\right)}^{2}\right)}}\right)
$$

For the CB we have that the general form for random variable $x$ and a fixed threshold $\gamma$ is $P(X>\gamma) \leq \mathrm{E}\{\exp [s(X-\gamma)]\}, s>0$. Thus $P(X>\gamma) \leq M_{X}(s) e^{-s \gamma}$, and manipulation of this for the difference of two random variables implies that,

$$
P\left(X_{0}\left(\Delta t_{j}\right)>X_{1}(\Delta t)\right) \leq M_{X_{1}(\Delta t)}(-s) M_{X_{0}\left(\Delta t_{j}\right)}(s) \quad(s>0)
$$

For the MCB [2], $P(X>\gamma) \leq M_{X}(s) e^{-s \gamma} / s \sigma_{t h} \sqrt{\pi}$. Modifying this inequality for the difference of two random variables for $x_{0}\left(\Delta t_{j}\right)$ and $x_{1}(\Delta t)$ which both have the same thermal noise contribution then yields,

$$
P\left(X_{0}\left(\Delta t_{j}\right)>X_{1}(\Delta t)\right) \leq \frac{M_{X_{1}(\Delta t)}(-s) M_{X_{0}\left(\Delta t_{j}\right)}(s)}{2 s \sigma_{t h} \sqrt{\pi}} \quad(s>0)
$$

For the FA and OSA cases the symbol error probability in the presence of a specific crosstalk combination is written as,

$$
P_{\text {we }\left(l_{s-}-r_{s}\right)} \leq 1-\left(1-P\left(X_{0}(0)>X_{1}(\Delta t)\right)\right)^{n-1-\left(l_{s}-r_{s}\right)}\left(1-P\left(X_{0}\left(t_{s}\right)>X_{1}(\Delta t)\right)\right)^{l_{s}-r_{s}}
$$

where $l_{s}$ and $r_{s}$ are the number of crosstalk of duration $t_{s}$ occurring in the signal frame and signal pulse slot respectively, $\Delta t=t_{s}$ if crosstalk hits signal pulse slot, otherwise $\Delta t=0$.

Similarly, the symbol error probability in the presence of crosstalk for the SM case is written as,

$$
\left.P_{\text {we }\left(l_{1}, l_{2}-r_{1}, r_{2}\right)} \leq 1-\left(1-P\left(X_{0}(0)>X_{1}(\Delta t)\right)\right){ }^{n-1-\alpha}\left(1-P\left(X_{0}\left(t_{1}\right)>X_{1}(\Delta t)\right)\right){ }^{l_{1}-r_{1}}\left(1-P\left(X_{0}\left(t_{2}\right)>X_{1}(\Delta t)\right)\right)\right)^{l_{2}-r_{2}}
$$


where $l_{1}, l_{2}$ and $r_{1}, r_{2}$ are the number of crosstalk of duration $t_{1}, t_{2}$ occurring in the signal frame and signal pulse slot respectively, $\alpha=l_{1}+l_{2}-r_{1}-r_{2}, \Delta t=t_{1}$ or $t_{2}$ if crosstalk of duration $t_{1}$ or $t_{2}$ respectively hits the signal pulse slot, otherwise $\Delta t=0$. Note that in writing (10) part crosstalk pulses are counted. So, for example, a whole crosstalk pulse in the frame will nevertheless count as a unit contribution to both $l_{1}$ and $l_{2}$.

\section{BER Analysis (single crosstalk)}

For a single interferer, only one crosstalk pulse can hit the signal slot or an empty slot, although more than one crosstalk pulse can impair the signal frame if there is a misalignment between the signal and crosstalk frames. Clearly, for FA and OSA, only a full crosstalk pulse with overlap duration $\Delta t=t_{s}$ may occur. Let $p_{f\left(l_{s}\right)}\left(n_{1}\right)$ denote the probability of $l_{s}$ crosstalk pulses hitting the signal frame where $n_{1}$ is the number of whole slots in crosstalk frame 1 that overlap the signal frame. Also let $p_{s\left(l_{s}\right)}\left(r_{s}\right)$ denote the probability of $r_{s}$ out of $l_{s}$ crosstalk pulses hitting the signal slot so that the probability that a full crosstalk pulse hits the signal pulse slot $p_{s\left(l_{s}\right)}(1)=l_{s} / n$ and the probability that full crosstalk pulse(s) hit an (unspecified) empty slot $p_{s\left(l_{s}\right)}(0)=\left(n-l_{s}\right) / n$. Furthermore, once there is slot misalignment (SM), any or both partial crosstalk pulse(s) with overlap durations $\Delta t=t_{1}$ and $\Delta t=t_{2}$ (where $t_{s}=t_{1}+t_{2}$ ) could occur. Thus, $p_{f\left(l_{1}, l_{2}\right)}\left(n_{1}\right)$ denote the probability of $l_{1}$ and $l_{2}$ crosstalk pulses hitting the signal frame and $p_{s\left(l_{1}, l_{2}\right)}\left(r_{1}, r_{2}\right)$ denote the probability of $r_{1}$ out of $l_{1}$ and $r_{2}$ out of $l_{2}$ crosstalk pulses hitting the signal slot. 


\section{Frames Aligned (FA)}

Since there is only one pulse in a frame, it may be seen in Fig. 2a that for FA only one full crosstalk pulse can impair the signal frame and $p_{f(1)}(n)=1$ (seen from the special case of $p_{f(1)}\left(n_{1}\right)=\left(n_{1}^{2}+\left(n-n_{1}\right)^{2}\right) / n^{2}$ with $n_{1}=n$ in OSA below). As every frame's pulse has equal likelihood of being in any slot, the probability that a crosstalk pulse hits the signal slot for FA $p_{s(1)}(1)=1 / n$, and the probability that a crosstalk pulse hits an empty slot $p_{s(1)}(0)=(n-1) / n$.

The overall BER in the presence of crosstalk for frames (and slots) aligned is given as [28],

$$
B E R=\frac{n}{2(n-1)}\left(p_{s(1)}(1) P_{w e(1-1)}+p_{s(1)}(0) P_{w e(1-0)}\right)
$$

\section{Only Slots Aligned (OSA)}

Once there is a misalignment (whether frames only or frames and slots), it is possible for zero, one or two crosstalk pulses to impair the signal frame. Fig $2 \mathrm{~b}$ shows a typical example of how two crosstalk from a single interferer can impair the signal frame, however, these two crosstalk can only be in different slots in the signal frame as only one crosstalk can hit a slot for single crosstalk case. If the pulse in crosstalk frame1 in Fig. $2 \mathrm{~b}$ was transmitted much earlier in that frame instead, there will be only one crosstalk impairing the signal frame. Furthermore, if additionally the pulse in crosstalk frame 2 in Fig. $2 \mathrm{~b}$ was transmitted later, there will be no crosstalk impairing the signal frame.

Using Fig. 2b, with $n_{1}+n_{2}=n$, the occurrence probabilities $p_{f\left(l_{s}\right)}\left(n_{1}\right)$ for the three different possibilities of a hit on the signal frame are found as $p_{f(0)}\left(n_{1}\right)=p_{f(2)}\left(n_{1}\right)=n_{1}\left(n-n_{1}\right) / n^{2}$ and $p_{f(1)}\left(n_{1}\right)=\left(n_{1}^{2}+\left(n-n_{1}\right)^{2}\right) / n^{2}$. Also the bit error rate contributions for the different possibilities are conditional on $n_{1}$ and generally written as, 


$$
\operatorname{BER}_{l_{s}}\left(n_{1}\right)=p_{f\left(l_{s}\right)}\left(n_{1}\right) \frac{n}{2(n-1)}\left(p_{s\left(l_{s}\right)}(1) P_{w e\left(l_{s-1}\right)}+p_{s\left(l_{s}\right)}(0) P_{w e\left(l_{s-0}\right)}\right)
$$

with the no crosstalk symbol error probability $P_{\text {we }\left(0_{-} 0\right)}$ treated the same as in $[1,2]$, and

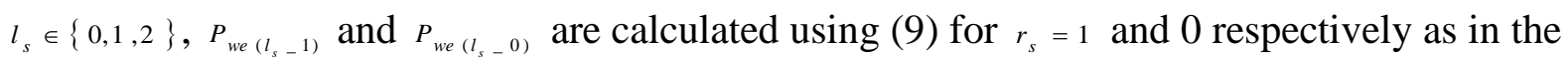
FA case. However, since there could be two crosstalk in the signal frame under OSA, the overall BER in the presence of crosstalk for only slots aligned is calculated by summing up all the error contribution calculated from (12) for all values of $l_{s}$ and the conditioning on $n_{1}$ is removed through averaging (assuming signal and crosstalk walk off each other sufficiently fast). It is written as

$$
B E R=\frac{1}{n} \sum_{n_{1}=1}^{n} \sum_{l_{s}=0}^{2} B E R l_{l_{s}}\left(n_{1}\right)
$$

\section{Slots Misaligned (SM)}

The number of different crosstalk combinations occurring in the signal frame increases with slot misalignment, with the detailed analysis becoming complicated. Considering Fig. 2c, with $n_{1}+n_{2}=n+1$ (note the difference to the OSA case), there are seven different crosstalk possibilities each with different occurrence probability $p_{f\left(l_{1}, l_{2}\right)}\left(n_{1}\right)$ calculated from a given $n_{1}$ (contributing to the overall symbol error probability) regarding how much crosstalk hits a frame as follows:

i) No crosstalk in the signal frame with probability $p_{f(0,0)}\left(n_{1}\right)=\left(n_{1}-1\right)\left(n-n_{1}\right) / n^{2}$ : e.g. as shown when the pulses in XT frame 5 and frame 6 respectively occur before the signal frame 5 begins and after the signal frame 5 ends. 
ii) Only one $t_{1}$ partial crosstalk pulse in the signal frame with probability $p_{f(1,0)}\left(n_{1}\right)=\left(n_{1}-1\right) / n^{2}:$ e.g. as shown when XT frame 2 pulse occurs at signal frame 1 end while the XT frame 1 pulse occurs before the start of signal frame 1.

iii) Only one $t_{2}$ partial crosstalk pulse in the signal frame with probability $p_{f(0,1)}\left(n_{1}\right)=\left(n-n_{1}\right) / n^{2}$ : e.g. as shown when XT frame 3 pulse occurs after signal frame 2 ends while the XT frame 2 pulse occurs at the start of signal frame 2.

iv) One each of $t_{1}$ and $t_{2}$ partial crosstalk pulse in the signal frame with occurrence probability $p_{f(1,1)}\left(n_{1}\right)=\left((n-1)^{2}+1\right)-\left(2\left(n_{1}-1\right)\left(n-n_{1}\right)\right) / n^{2}$ : e.g. as shown when XT frame 6 pulse occurs within signal frame 6. The other possibilities (not shown) are, (a) when XT frame 7 pulse occurs within signal frame 6 and (b) where each of XT frames 6 and 7 contribute a part pulse at the start and end of signal frame 6 respectively.

v) One $t_{1}$ and two $t_{2}$ partial crosstalk pulses in the signal frame with probability $\left(p_{f(1,2)}\left(n_{1}\right)=\left(n_{1}-1\right) / n^{2}\right)$ : e.g. as shown when whole XT pulse from XT frame 5 occurs within signal frame 4 and XT frame 4 pulse occurs at the start of signal frame 4.

vi) Two $t_{1}$ and one $t_{2}$ partial crosstalk pulses in the signal frame with probability $\left(p_{f(2,1)}\left(n_{1}\right)=\left(n-n_{1}\right) / n^{2}\right)$ : e.g. as shown when whole XT pulse from XT frame 3 occurs in signal frame 3 and XT frame 4 pulse occurs at the end of signal frame 3.

vii) Two each of $t_{1}$ and $t_{2}$ partial crosstalk pulses in the signal frame with occurrence probability $\left(p_{f(2,2)}\left(n_{1}\right)=\left(n_{1}-1\right)\left(n-n_{1}\right) / n^{2}\right)$ : e.g. as shown when whole XT pulses from both XT frames 7 and 8 occur within signal frame 7. 
Also, at the slot level for the SM, the probabilities that partial crosstalk pulses of duration $\Delta t=t_{1}$ and $\Delta t=t_{2}$ hit the signal slot are $p_{s\left(l_{1}, l_{2}\right)}(1,0)=l_{1} / n$ and $p_{s\left(l_{1}, l_{2}\right)}(0,1)=l_{2} / n$ respectively, and for a hit on empty slot, $p_{s\left(l_{1}, l_{2}\right)}(0,0)=\left(n-l_{1}-l_{2}\right) / n$.

Assuming the slot is discretized into $m$ small units of length $t_{c}=t_{s} / m$ such that the minimum slot offset equals $t_{c}$, then $t_{1}$ takes values from $\left\{t_{c}, 2 t_{c}, 3 t_{c} \ldots m t_{c}\right\}$ where $t_{1}+t_{2}=t_{s}=m t_{c}$. For definiteness, $m=100$ is used in the calculations in this paper as higher values of $m$ do not show any significant effect on the results, but rather increases the computational time. The OSA case is recovered for $m=1$.

The bit error rate contribution when there is no crosstalk is written as,

$$
\operatorname{BER}\left(n_{1}\right)=p_{f(0,0)}\left(n_{1}\right) \frac{n}{2(n-1)} P_{\text {we }(0,0-0,0)}
$$

while for the other possibilities, it is generally written as :

$$
\begin{aligned}
\operatorname{BER} l_{l_{1}, l_{2}}\left(n_{1}\right)=\frac{1}{m} \sum_{t_{1}=t_{c}}^{t_{s}} & p_{f\left(l_{1}, l_{2}\right)}\left(n_{1}\right) \frac{n}{2(n-1)} \\
& \left(p_{s\left(l_{1}, l_{2}\right)}(1,0) P_{\text {we }\left(l_{1}, l_{2}-1,0\right)}+p_{s\left(l_{1}, l_{2}\right)}(0,1) P_{\text {we }\left(l_{1}, l_{2}-0,1\right)}+p_{s\left(l_{1}, l_{2}\right)}(0,0) P_{w e}\left(l_{1}, l_{2}-0,0\right)\right.
\end{aligned}
$$

The no crosstalk symbol error probability $P_{\text {we }\left(0,0_{-}, 0,0\right)}$ is calculated the same as $P_{\text {we }\left(0_{-} 0\right)}$ in the SA case, and $l_{1}, l_{2} \in\{0,1,2\}$ excluding the case where $\left(l_{1}, l_{2}\right)=(0,0)$. The other symbol error probabilities $P_{\text {we }\left(l_{1}, l_{2}-1,0\right)}, P_{\text {we }\left(l_{1}, l_{2}-0,1\right)}$, and $P_{\text {we }\left(l_{1}, l_{2}-0,0\right)}$ are calculated using equation (10) for $\left(r_{1}, r_{2}\right)=(1,0),(0,1)$ and $(0,0)$.

The overall BER in the presence of crosstalk for slots misaligned is calculated by summing up all the error contributions calculated from (14) and (15) with the conditioning on $n_{1}$ removed by averaging. It is written as 


$$
B E R=\frac{1}{n} \sum_{n_{1}=1}^{n}\left\{B E R\left(n_{1}\right)+B_{0,1}\left(n_{1}\right)+\operatorname{BER}_{1,0}\left(n_{1}\right)+\sum_{l_{1}=1}^{2}\left[\operatorname{BER}_{l_{1}, 1}\left(n_{1}\right)+\operatorname{BER}_{l_{1}, 2}\left(n_{1}\right)\right]\right\}
$$

\section{Single Crosstalk Results}

The system parameters used in the model are listed in Table $1 . N_{o_{-} X T}$ is fixed by $N_{o} / R_{X T}$ at the receiver with $R_{X T}>1$, i.e. assuming that the crosstalk and the accompanying ASE have been attenuated by the demultiplexer upon coupling to the desired signal photodetector. The same data rate is assumed for both crosstalk and signal. The DPPM thermal noise variance is back calculated using a bandwidth expansion factor such that $\sigma_{t h-D P M}^{2}=B_{\text {exp }} \sigma_{\text {th-OoK }}^{2}$ where $B_{\text {exp }}=2^{M} / M$ is the DPPM bandwidth expansion factor [29] and $\sigma_{t h-o o K}=7 \times 10^{-7} \mathrm{~A}$ is obtained from a model of a pinFET receiver with $R_{b}=2.5 \mathrm{Gbps}$ at BER of $10^{-12}$ assuming a sensitivity of $-23 \mathrm{dBm}$ [19]. The demux (or OBPF) channel bandwidth is $76 \mathrm{GHz}$ with $100 \mathrm{GHz}$ adjacent channel spacing, this is about the same with those seen in $[30,31]$ and will easily accommodate the slot rate of $45.7 \mathrm{GHz}$ for maximum DPPM coding level of $M=7$ considered [2]. Typical values for adjacent channel rejection ratio ranges from $-20 \mathrm{~dB}$ to $-30 \mathrm{~dB}$ [30-32], however in this work, the level of crosstalk (relative to signal at the photodiode) which could be worsened by asymmetric demux input powers is allowed to vary from negligible case of - $30 \mathrm{~dB}$ to a very worse case of $-5 \mathrm{~dB}$ and the resulting crosstalk effect is calculated and shown for each case. A target BER of $10^{-9}$ is considered for systems without forward error correction coding (FEC) $[33,34]$, and $10^{-3}$ is considered for systems with FEC. Also the required optical power is defined as the average power at the input of the optical amplifier required to achieve the target BER. 
Table 2: Physical parameters used for calculations

\begin{tabular}{|c|c|c|}
\hline Parameters & Description & Value \\
\hline$R_{b}$ & binary data rate & $2.5 \mathrm{Gbps}$ \\
\hline$B_{o p t}$ & demux channel optical noise bandwidth & $76 \mathrm{GHz}$ \\
\hline$\lambda_{\text {sig }}$ & signal wavelength & $1550 \mathrm{~nm}$ \\
\hline$\eta$ & receiver quantum efficiency & $0.9[35]$ \\
\hline G & optical preamplifier gain & $27 \mathrm{~dB}$ or $8 \mathrm{~dB}$ \\
\hline$N F$ & optical preamplifier noise figure & $4.77 \mathrm{~dB}[2]$ \\
\hline$m_{t}$ & ASE noise polarisation states & 2 \\
\hline
\end{tabular}

Fig. 3 shows the single crosstalk BER curves using the MCB technique for FA, OSA, SM and no crosstalk cases with high signal to crosstalk ratio $\left(R_{X T}=10 \mathrm{~dB}\right)$ and moderate signal to crosstalk ratio $\left(R_{X T}=5 \mathrm{~dB}\right)$ at low coding level $(M=1)$ and relatively high coding level $(M=5)$ . The curves for the GA and CB techniques are similar, just offset by less than $0.3 \mathrm{~dB}$ at a BER of $10^{-9}$.

The BER for FA case is seen to exceed all other cases in all the methods considered and thus results in the worst case power penalty. The OSA BER coincides with the FA BER at $M=5$, while the SM case produces the best BER curves at all coding levels. The similarity between the OSA and the FA (which also is a special and dominant subcase of the OSA, occurring at maximum overlap of a particular crosstalk frame with the signal frame) can be understood from 
the probabilities of the OSA crosstalk distribution. For example, at minimum overlap of frame 1 in Fig. $2 \mathrm{~b}, n_{1}=1$ and the OSA probabilities are dominated by the probability of one crosstalk hitting the signal frame $p_{f(1)}=\left(1+(n-1)^{2}\right) / n^{2}>\left(p_{f(0)}=p_{f(2)}=(n-1) / n^{2}\right)$. It is easily seen that as $n$ gets larger, $p_{f(1)} \rightarrow 1$ while $p_{f(0)}=p_{f(2)} \rightarrow 0$ and OSA approximates to FA.
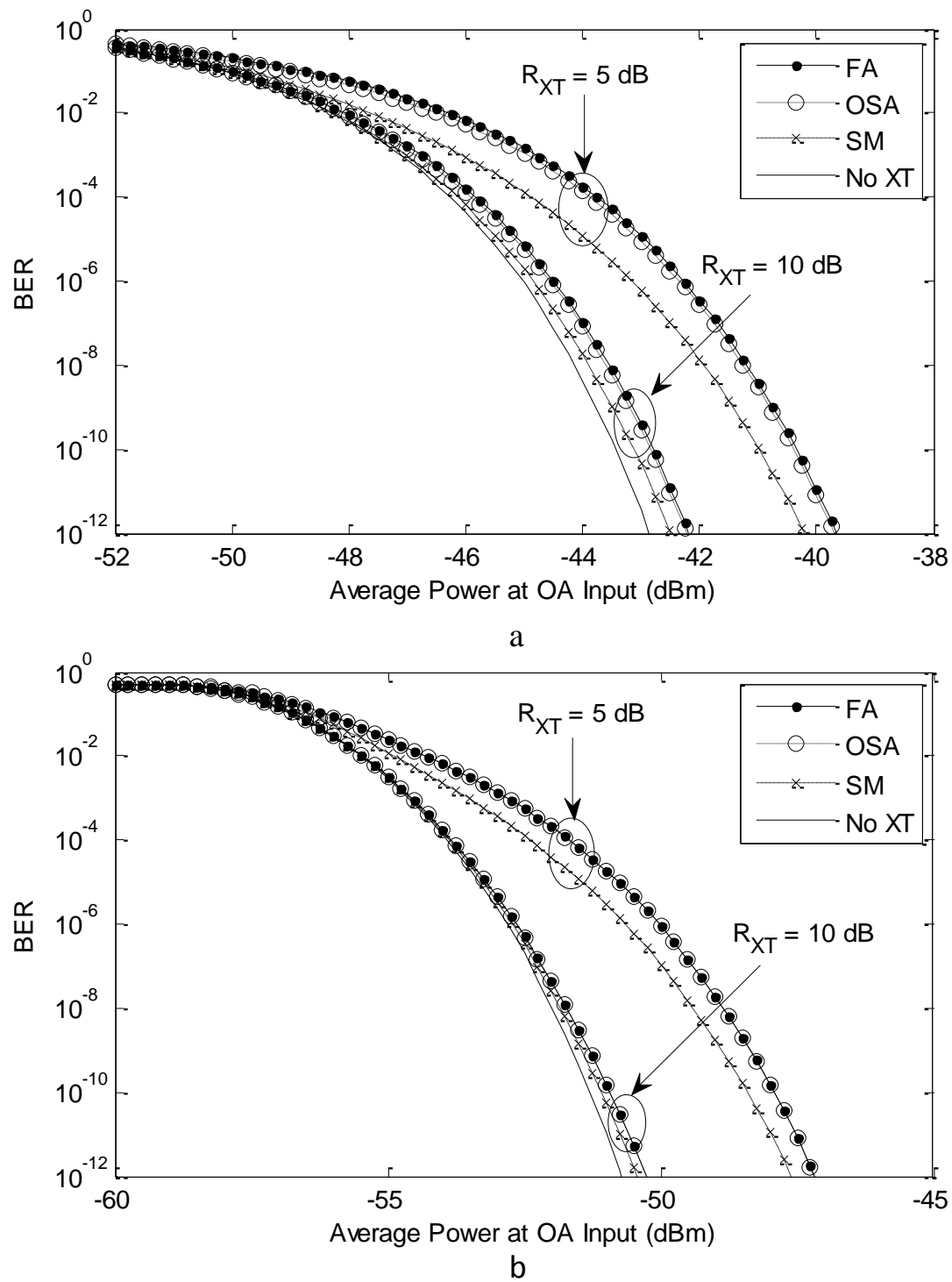

Fig. 3: BER against average power at $\mathrm{OA}$ input $(\mathrm{dBm})$ using $\mathrm{MCB}, G=27 \mathrm{~dB}$, for 1 crosstalk $-R_{X T}=10 \mathrm{~dB}$ and $5 \mathrm{~dB}$ (a) $M=1$ (b) $M=5$

Fig. 4 shows a comparison of the GA, CB and MCB performance at low gain $G=8 \mathrm{~dB}$ and high gain $G=27 \mathrm{~dB}$ with a single crosstalk source and $M=2$. The MCB coincides with the GA at 
low gain, but shifts close to the $\mathrm{CB}$ at high gain as the ASE noise reduces the significance of the thermal noise. The GA on the other hand is seen to exceed the $\mathrm{CB}$ and MCB (which are upper bounds) at high gain with no crosstalk and in the presence of crosstalk. The margin with which the GA exceeds the MCB and $\mathrm{CB}$ widens as the coding level and the noise equivalent bandwidth $B_{e}$ of the DPPM receiver increases. This inconsistent behaviour of the GA is well reported for both OOK and DPPM systems[2, 26], but it has the advantage of being a simple and quick performance evaluation technique.
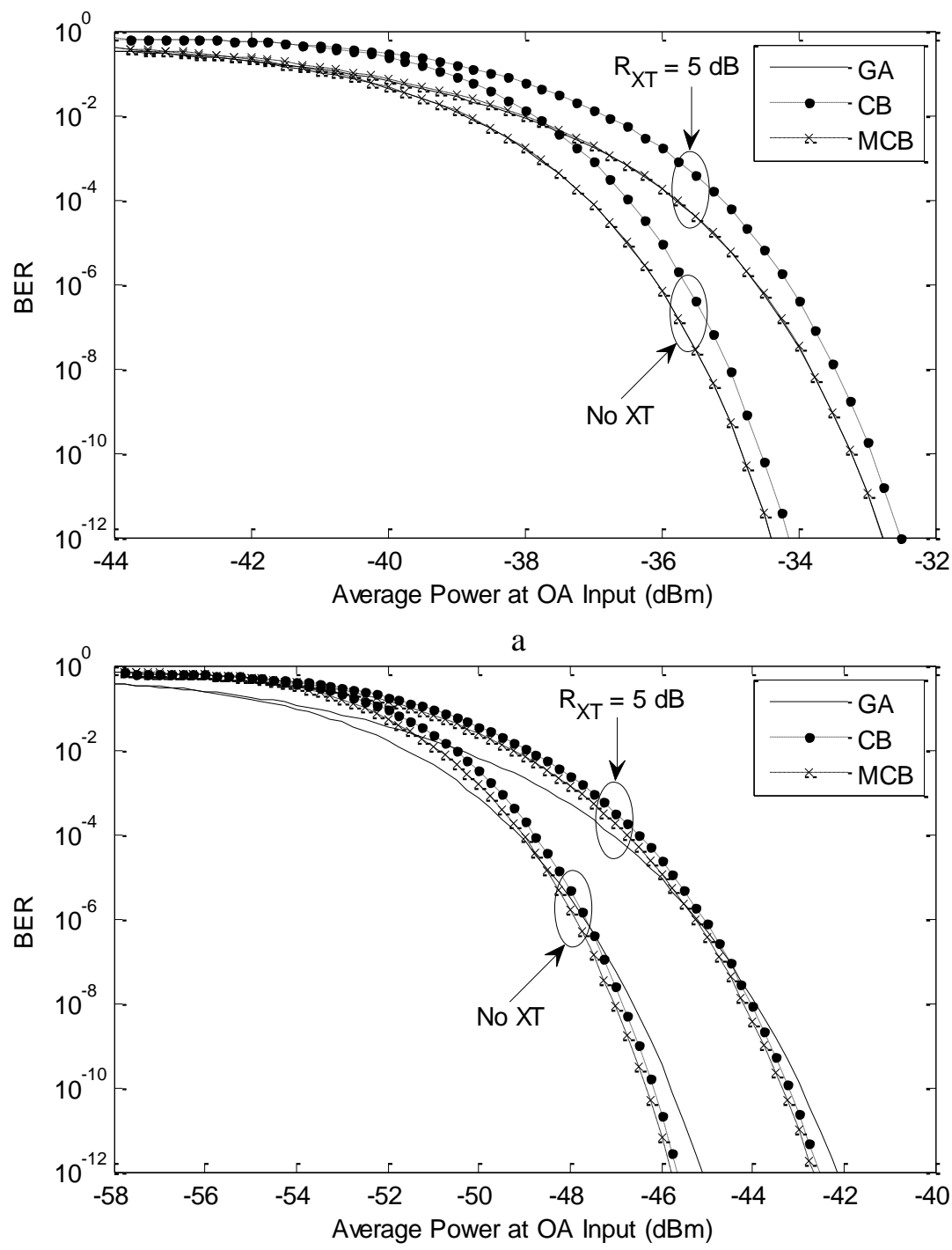

b

Fig. 4: BER against average power at $\mathrm{OA}$ input $(\mathrm{dBm})$ using $M=2$, for FA single crosstalk with $R_{X T}=5 \mathrm{~dB}$ (a) $G=8 \mathrm{~dB}$ (b) $G=27 \mathrm{~dB}$ 
To further understand the single crosstalk system, consider Fig. 5 which shows the result of power penalty as a function of fixed misalignment. Each point in Fig. 5a presents the power penalty for the different fixed slot alignments (subcases) that is averaged to obtain the overall power penalty for the OSA case The penalty at $n_{1}=8$ corresponds to the penalty for FA. The best performance for the fixed slot alignments is attained at $n_{1}=4$, this is because the probability of no crosstalk impairing the signal frame $p_{f(0)}\left(n_{1}\right)$ is highest for such misalignment. Fig. 5b presents the power penalty for fixed frame and slot misalignment SM and gives a better insight of a more practical system. The result highlights the importance of the averaging approach for realistic systems as recommended earlier. All the points in Fig $5 b$ are averaged to obtain the overall power penalty for the SM case as per (16). The points along the $n_{1}$ axis at $t_{1}=1$ are the fixed slot alignment points and are the same as the result presented in Fig. 5a. The FA point occurs at $n_{1}=8, t_{1}=1$, and is seen to present the worst penalty. Optimum points also occur along the $t_{1}$ axis at $t_{1}=0.5$ and implicitly, at $t_{2}=0.5$. This is because the maximum power of either partial crosstalk i.e. $\max _{\left\{P_{t_{1}}, P_{t_{2}}\right\}}$ is lowest at that point. On the left of this optimum, $t_{2}>0.5$ and on the right $t_{1}>0.5$. Thus, it is clearly seen that the impact of a single high power crosstalk is worse than that of many crosstalk of equivalent power. The result in Fig. 5 could be of practical importance in a non-dispersive channel like in free space where fixed misalignment may persist for a longer duration that averaging may not be required. 

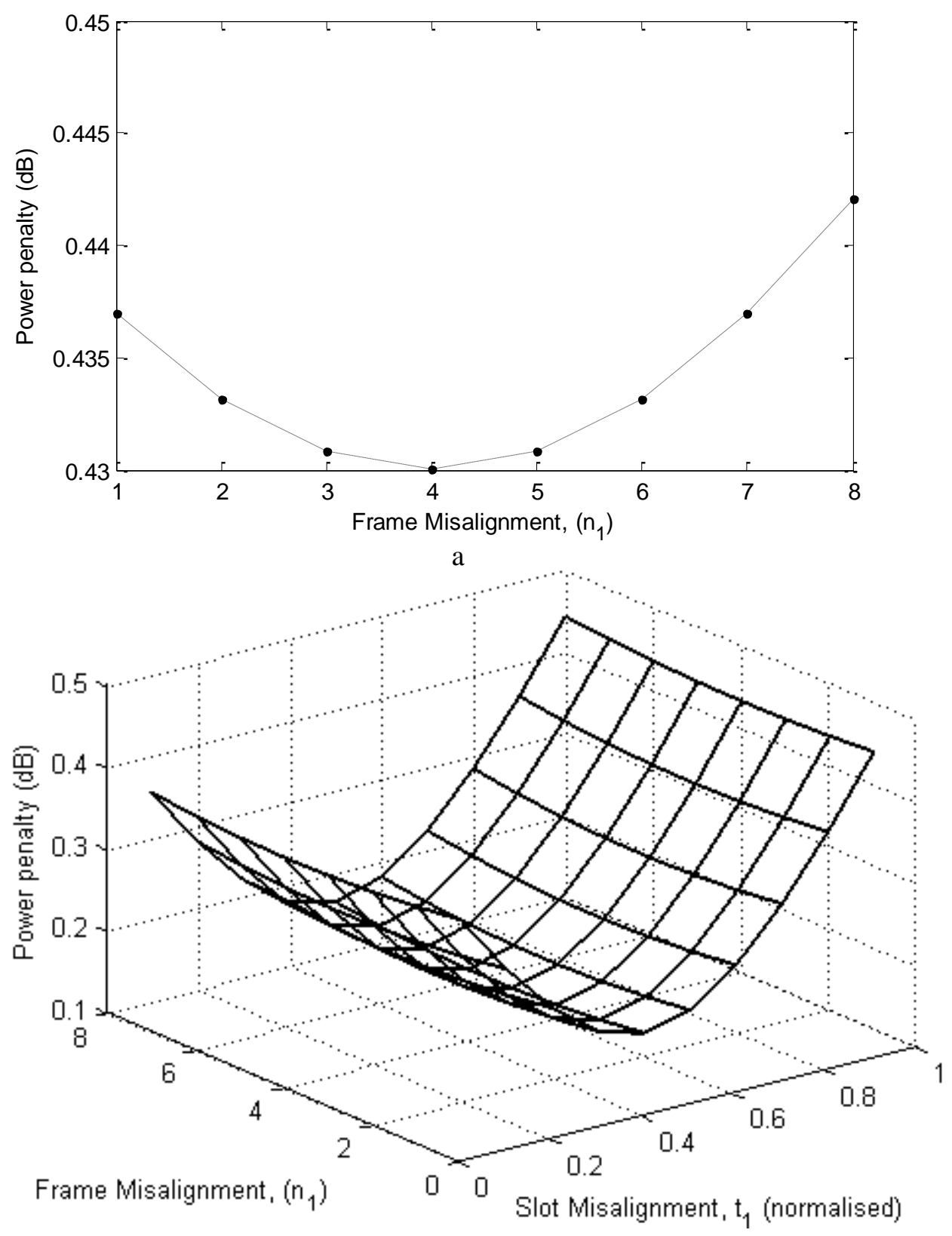

b

Fig. 5: Power penalty as a function of fixed misalignment using MCB (single crosstalk) for $M$ $=3$ and $R_{X T}=10 \mathrm{~dB}$ at BER $=10^{-9}$ (a) Frame misalignment in OSA case (b) Frame and slot misalignment in SM case

The remaining results for single crosstalk analysis are obtained assuming the FA case (which has just been shown as the worst case performance).

In Fig. 6, the result of the required signal power and the power penalty as a function of DPPM coding level and signal to crosstalk ratio using the MCB is shown. The required signal power 
in Fig. 6a is seen to decrease as the coding level increases for all values of signal to crosstalk ratio, but at each coding level, the required signal power increases as the crosstalk power increases. The same pattern is also seen in Fig. $6 \mathrm{~b}$ with the power penalty increasing as the crosstalk power increases for each coding level. The ideal OOK power penalty (i.e. with extinction ratio $r \rightarrow \infty$ ) coincides with the DPPM power penalty for $M=1$ as shown in Fig. 6c. However, the DPPM becomes better than OOK as the coding level increases. 

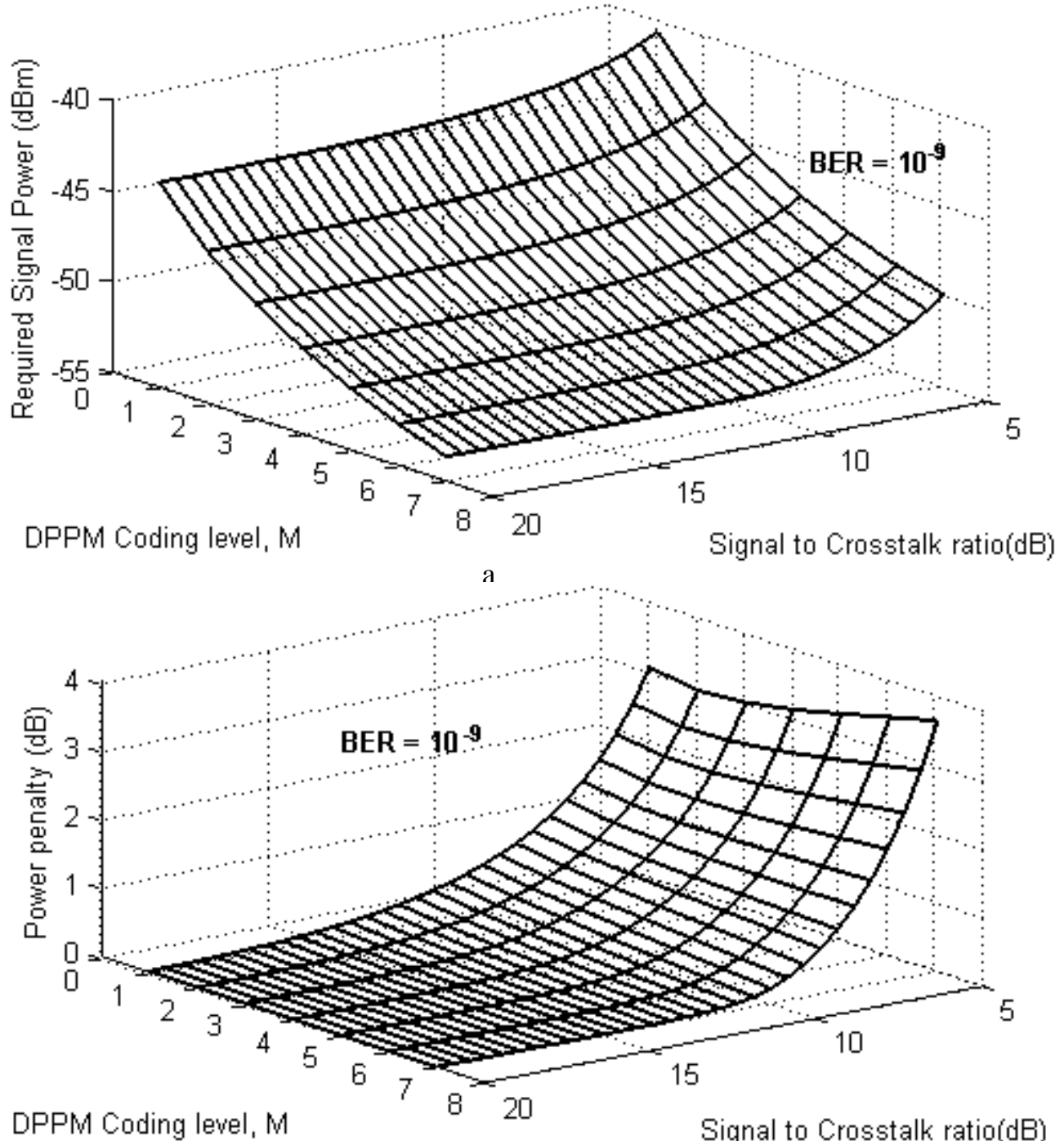

b

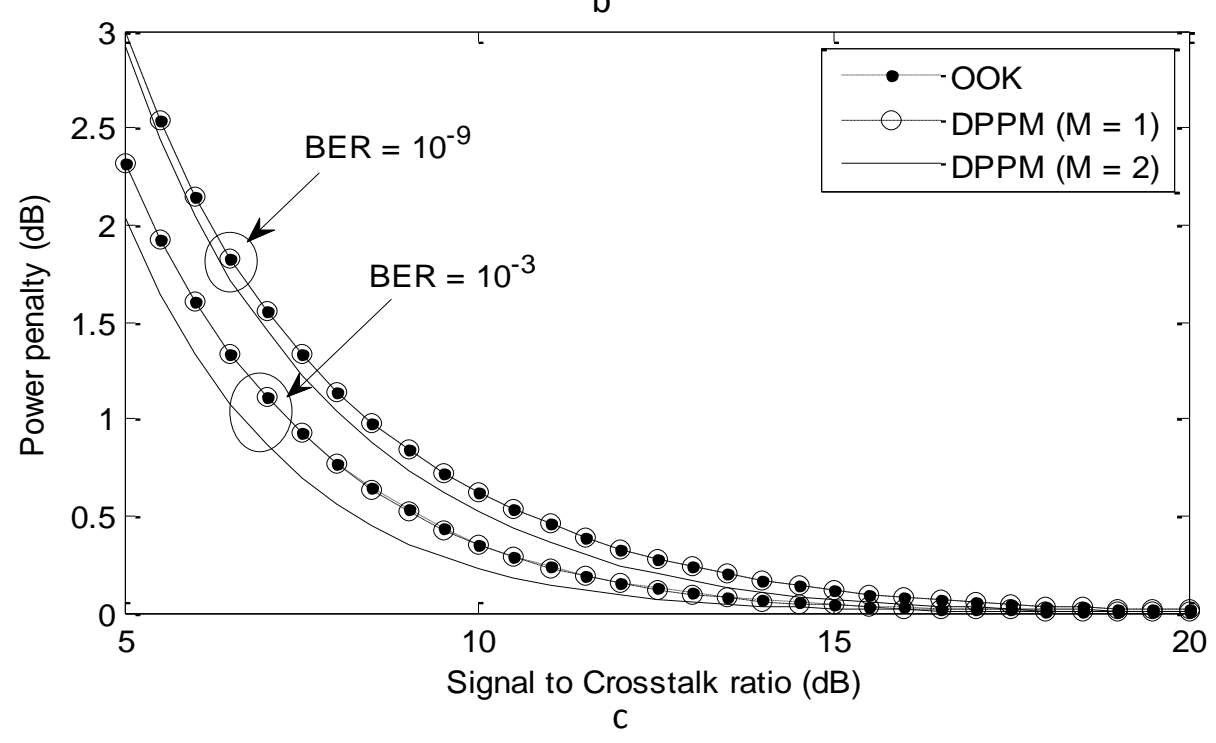

Fig. 6: DPPM coding level and Signal-to-crosstalk ratio against Required signal power and Power penalty using MCB (FA single crosstalk) (a) Required signal power (b) Power penalty (c) DPPM compared with OOK (Power penalty vs. Signal-to-crosstalk ratio) 


\section{BER Analysis (multiple crosstalk)}

For a large number of crosstalk arising from different wavelength channels in the system, the analysis under the constraint of slot or frame misalignment could be very complex and computationally intensive. It could require examining the content of each slot under every possible misalignment of slots and/or frame for all the crosstalk in order to determine their occurrence probabilities and symbol error probability contributions. However, the assumption of all frames aligning (FA) (acceptable as argued previously for a single crosstalk) becomes less likely with increasing $N$ such that imposing such constraint overestimates the BER or power penalty for large $N$ values. Therefore the only slots aligned (OSA) approach seems to be the most sensible for multiple crosstalk as it is also much quicker than the SM approach.

The multiple crosstalk analysis is considered for $M=1$ and $M=2$, which are more practical cases for WDM systems and the analysis is facilitated by the GA for computational ease. The probabilities for the OSA approach have been validated by Monte Carlo simulation and are presented analytically only for $M=1$. For simplicity, the probability of crosstalk distribution for $M=2$ is generated by Monte Carlo simulation. All the crosstalk pulses are assumed to have equal power. This is the case when there is symmetry in the transmission link. Alternatively, when the amounts of crosstalk in individual wavelengths are different or there is a single dominant crosstalk in the system it may be more convenient to add all the interfering crosstalk power together and treat the equivalent crosstalk power as if it is from a single wavelength using the single crosstalk model discussed earlier. This at least provides an upper bound for the crosstalk power penalty. 


\section{Frames Aligned (FA)}

Under FA and for $m_{1}=1$, there are only two slots in the frame and crosstalk pulses can either hit the signal slot or the empty slot. The probability that for ${ }_{N}$ crosstalk signals, $c$ of them hit the signal slot while $d=N-c$ of them hit an empty slot is defined by the binomial,

$$
p_{s}(c, d)=\left(\begin{array}{l}
N \\
c
\end{array}\right)\left(\frac{1}{2}\right)^{c}\left(\frac{1}{2}\right)^{d}
$$

and the overall BER in the presence of $N$ crosstalk pulses for $M=1$ is written as:

$$
B E R=\frac{n}{2(n-1)} \sum_{c=0}^{N} p_{s}(c, d)\left(1-\left(1-P\left(X_{0}\left(d t_{s}\right)>X_{1}\left(c t_{s}\right)\right)\right)\right)
$$

Also, for $M=2$, the probability distribution of crosstalk between the signal slot and the three empty slots is a binomial while the distribution of crosstalk within the three empty slots is a trinomial. The total probability distribution is a product of the binomial and trinomial distributions, written as:

$$
p_{s}\left(c, d_{1}, d_{2}, d_{3}\right)=\left(\begin{array}{l}
N \\
c
\end{array}\right) \frac{(N-c) !}{d_{1} ! d_{2} ! d_{3} !}\left(\frac{1}{4}\right)^{c}\left(\frac{1}{4}\right)^{N-c}
$$

and the overall BER in the presence of ${ }_{N}$ crosstalk pulses for $M=2$ is written as:

$$
\begin{aligned}
\text { BER }= & \frac{n}{2(n-1)} \sum_{c=0}^{N} \sum_{d_{1}, d_{2}, d_{3}=0}^{d} p_{s}\left(c, d_{1}, d_{2}, d_{3}\right) \\
& {\left[1-\left(1-P\left(X_{0}\left(d_{1} t_{s}\right)>X_{1}\left(c t_{s}\right)\right)\right)\left(1-P\left(X_{0}\left(d_{2} t_{s}\right)>X_{1}\left(c t_{s}\right)\right)\right)\left(1-P\left(X_{0}\left(d_{3} t_{s}\right)>X_{1}\left(c t_{s}\right)\right)\right)\right] }
\end{aligned}
$$

where $d_{1}, d_{2}, d_{3}$ are the number of crosstalk in empty slot 1,2,3 respectively, and $d_{3}=N-c-d_{1}-d_{2} \cdot X_{0}\left(d_{z} t_{s}\right)$ and $X_{1}\left(c t_{s}\right)$ are the random variables for empty slot $z$ hit by $d$ crosstalk pulses and signal slot hit by $c$ crosstalk pulses respectively. 


\section{Only Slots Aligned (OSA)}

The simplest method to generate the probability distribution of multiple crosstalk for the OSA case is by simulation, but for completeness, the analytical method is presented for $M=1$. With the OSA constraint, there is a chance that all, some, or no crosstalk frames align with the signal.

For $M=1$, and considering $N$ crosstalk with ${ }_{w}$ frames aligned with the signal frame, the total probability that $c$ and $d$ crosstalk pulses hit the signal slot and empty slot of the signal frame respectively is written as,

$$
p_{s}(c, d \mid w)=\frac{1}{n^{N}}\left(\begin{array}{l}
N \\
w
\end{array}\right)\left(\begin{array}{c}
w \\
k
\end{array}\right)\left(\begin{array}{c}
N-w \\
j
\end{array}\right)\left(\begin{array}{c}
N-w \\
j_{1}
\end{array}\right)\left(\frac{1}{2}\right)^{q}\left(\frac{1}{2}\right)^{2 N-w-q}
$$

where $q=k+j+j_{1}, c=k+j$ and $d=k_{1}+j_{1}$.

$k$ and $k_{1}$ are the number of crosstalk pulses from crosstalk with frames aligned with the signal frame that hit the signal slot and empty slot in the signal frame respectively and $j$ and $j_{1}$ are the number of crosstalk pulses from crosstalk with frames misaligned with the signal frame that hit the signal slot and empty slot in the signal frame respectively.

The overall BER in the presence of ${ }_{N}$ crosstalk pulses for $M=1$ is written as:

$$
B E R=\frac{n}{2(n-1)} \sum_{w=0}^{N} \sum_{k=0}^{w} \sum_{j, j_{1}=0}^{N-w} p_{s}(c, d \mid w)\left(1-\left(1-P\left(X_{0}\left(d t_{s}\right)>X_{1}\left(c t_{s}\right)\right)\right)\right)
$$




\section{Multiple Crosstalk Results}

Except where stated otherwise, the same parameters used for the single crosstalk model are maintained for the multiple crosstalk model. Also, the signal to crosstalk ratio $R_{X T}$ as used in the multiple crosstalk results refers to signal to single crosstalk ratio, arising as it does typically from the demultiplexer crosstalk rejection. The OOK model follows the same model for multiple crosstalk sources in [18] and with perfect extinction ratio assumed (so that any advantage of DPPM is not overstated).

The result of DPPM power penalty analyses for multiple crosstalk for $M=1$ and $M=2$ is compared with power penalty for OOK in Fig. 7 for target BER of $10^{-9}$. Clearly, DPPM predicts a reasonable penalty which is less than the OOK penalty for multiple crosstalk, even at low coding levels. The DPPM improvement in power penalty becomes better as the number of crosstalk sources increases and as the coding level increases from $M=1$ to $M=2$. In Fig. 7c, the FA is compared with OSA and simulation for $M=1$ and only simulation for $M=2$. Although the FA seems to overestimate the power penalty, the approximation gets better for ${ }_{M}=2$. Also, it is computationally quicker than the other approaches and provides an upper bound for the system. These same trends in Fig 7 are seen in Fig. 8, but with lower power penalties predicted for $10^{-3}$. This result is particularly of interest to modern high-sensitivity optical systems where FEC is commonly used. 


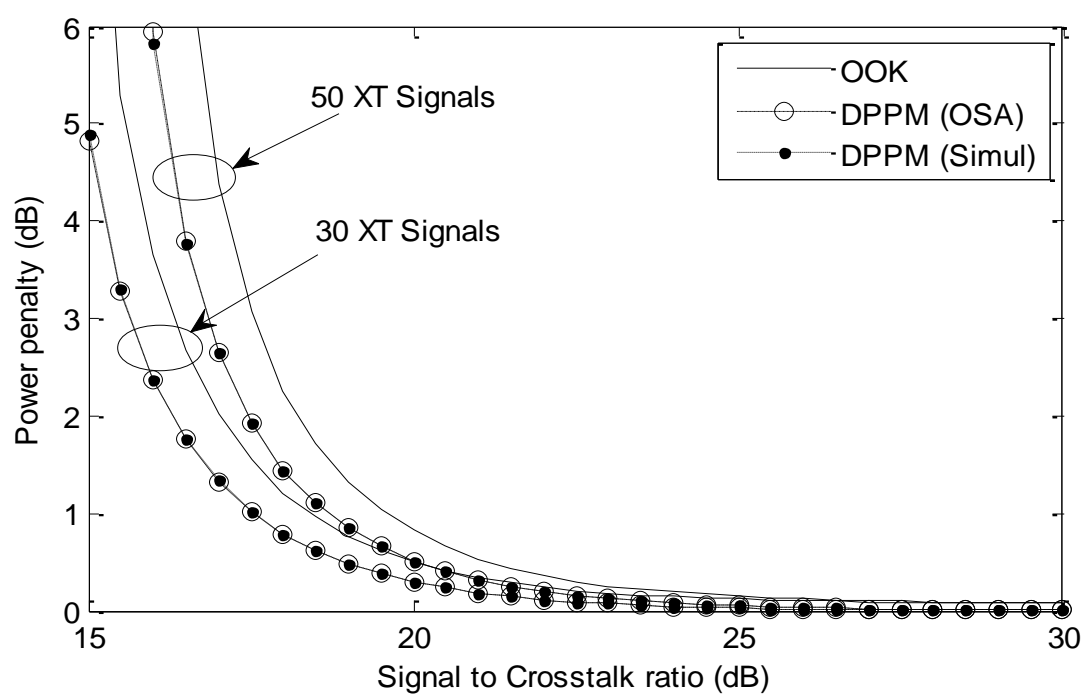

a

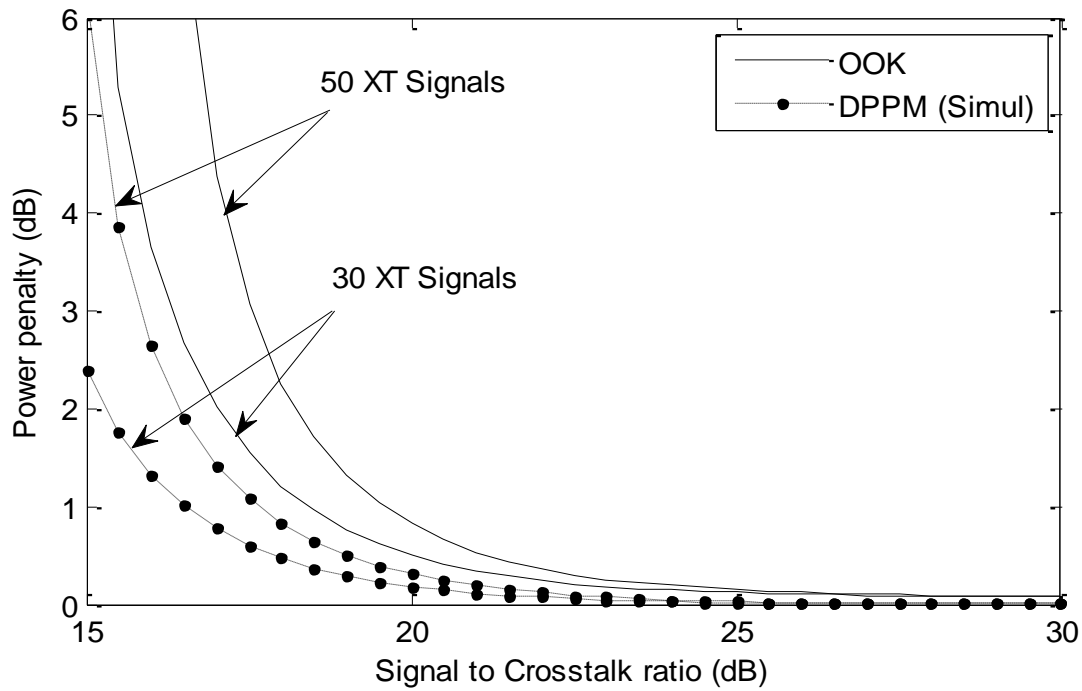

$\mathrm{b}$

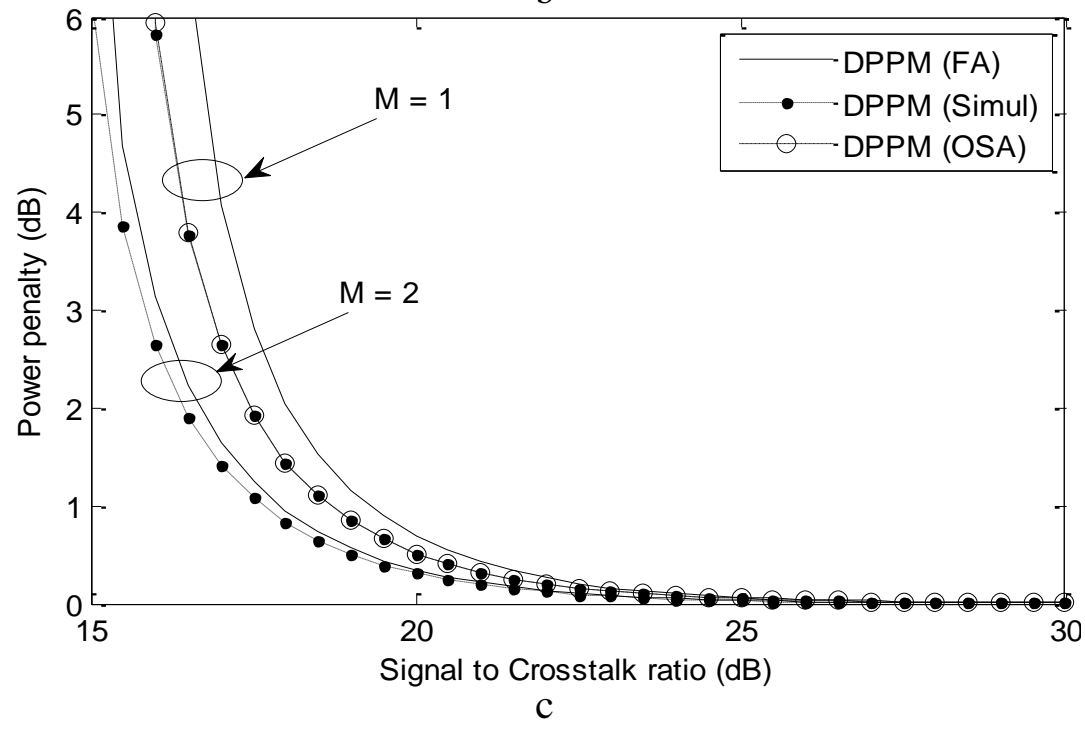

Fig. 7: Power penalty against Signal-to-crosstalk ratio for OOK and DPPM (multiple crosstalk OSA and Simulation) at BER $=10^{-9}$ (a) OOK comparison with DPPM at $M=1$ (b) OOK comparison with DPPM at $M=2$ (c) DPPM FA compared with OSA and/or Simulation (50 XT Signals) 


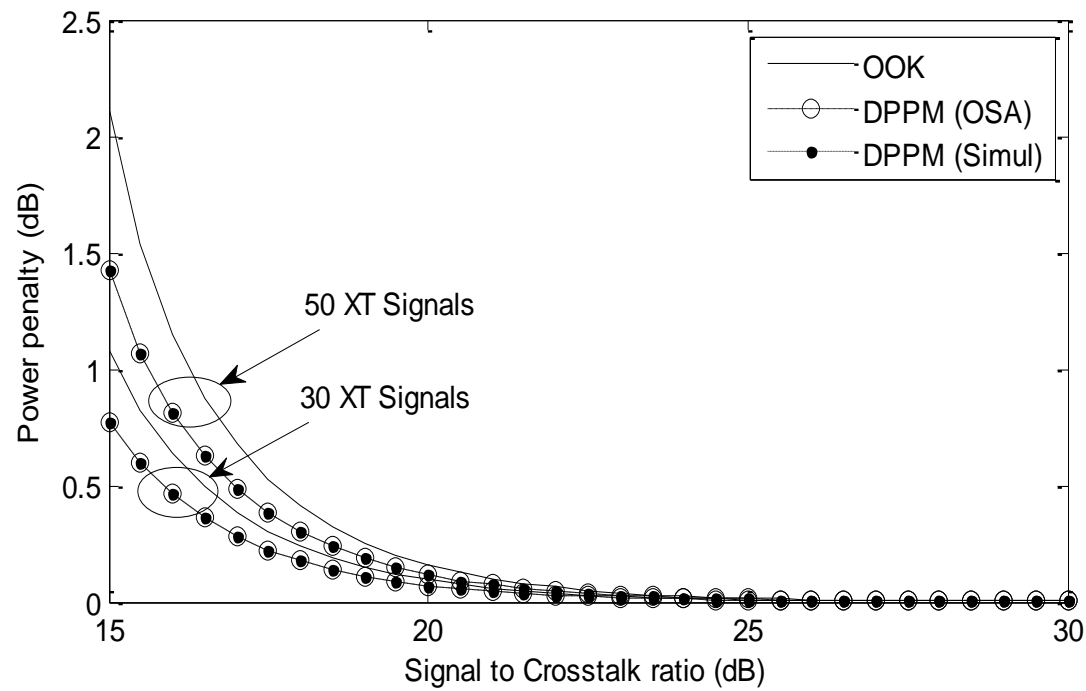

a

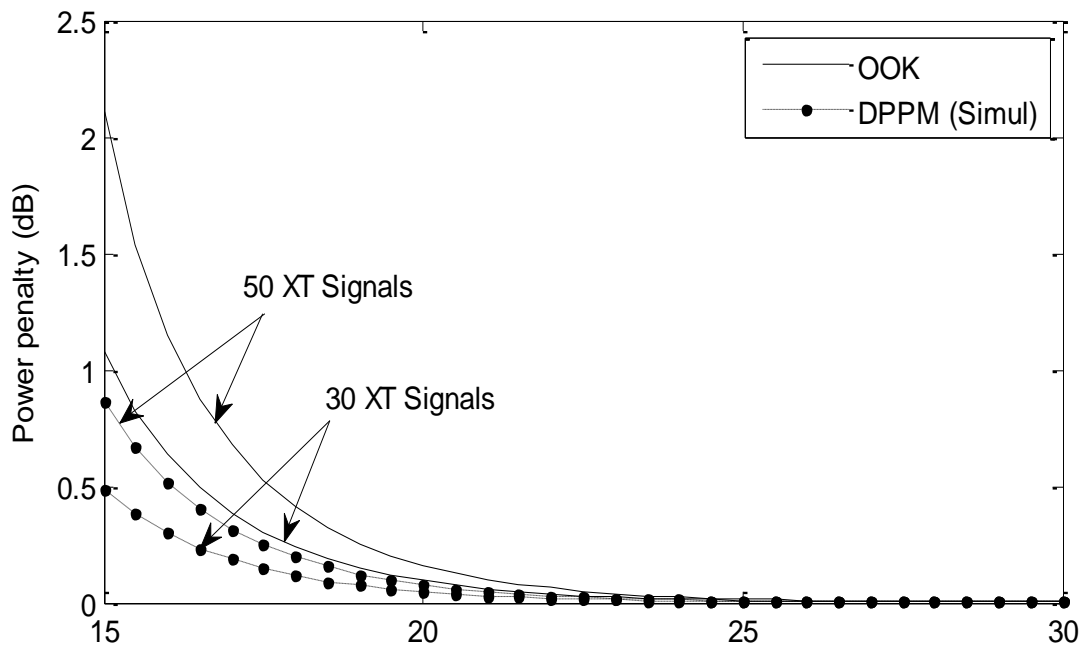

Signal to Crosstalk ratio $(\mathrm{dB})$

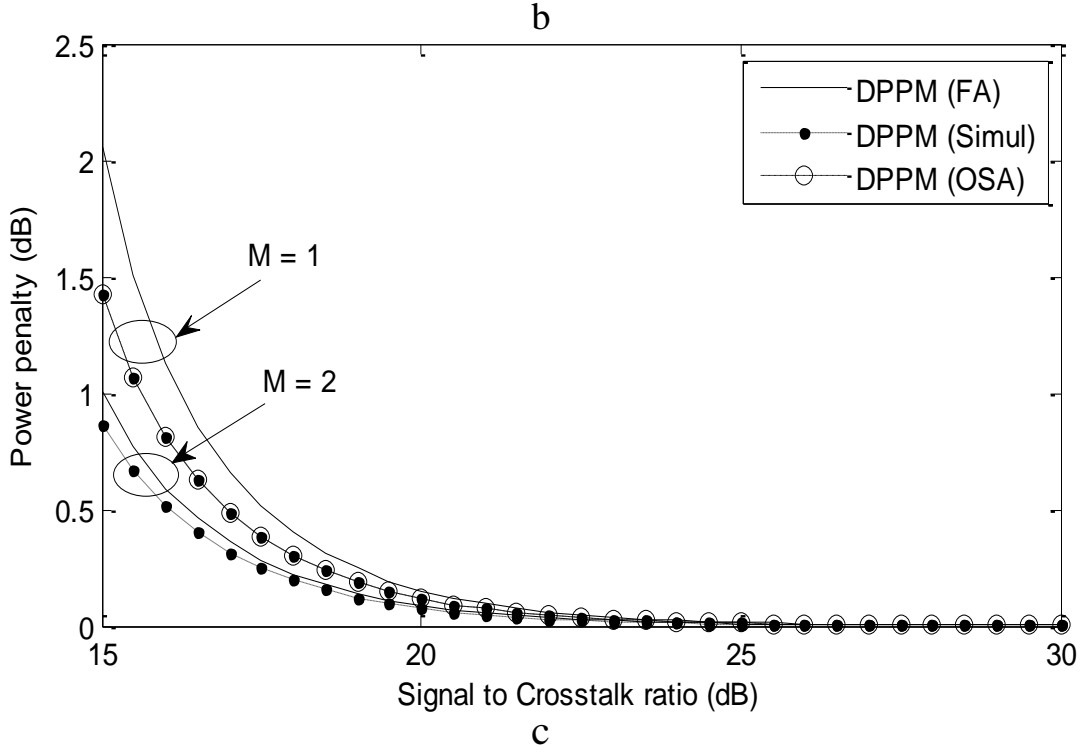

Fig. 8: Power penalty against Signal-to-crosstalk ratio for OOK and DPPM (multiple crosstalk OSA and Simulation) at BER $=10^{-3}$ (a) OOK comparison with DPPM at $M=1$ (b) OOK comparison with DPPM at $M=2$ (c) DPPM FA compared with OSA and/or Simulation (50 XT Signals) 


\section{Conclusion}

Analyses of crosstalk for optically preamplified WDM DPPM systems are performed for the first time using the GA, $\mathrm{CB}$ and MCB. The FA case is found to marginally present the worst power penalty. However the accuracy penalty is justified by a significant reduction in calculation complexity. For multiple crosstalk, the probability distribution of the crosstalk is easily obtained using Monte Carlo simulation. However for a fixed coding level, it is possible to analytically find the probability distribution of crosstalk in the signal frame by considering all the different multinomial contributions from every possible combination of aligned and misaligned crosstalk frames. The approach using the OSA assumption predicts a sensible penalty compared to the approach with FA assumption and hence presents a better representation of a practical system. Also, the MCB is recommended as the safest method of evaluation as it presents a tighter upper bound than the $\mathrm{CB}$ and is more sensitive to the optical amplification, though the GA is computationally quicker. The coding level with $M=2$ is a likely option for WDM DPPM free space and wireless systems because of its sensitivity improvement for a small bandwidth expansion over OOK, and when crosstalk is present this is further benefited by a reduced power penalty relative to OOK. 


\section{References}

1. Phillips, A. J., Cryan, R. A., Senior, J. M.: 'An optically preamplified intersatellite PPM receiver employing maximum likelihood detection'. IEEE Photonics Technol. Lett., 1996, 8, (5), pp. 691-693

2. Aladeloba, A. O., Phillips, A. J., Woolfson, M. S.: 'Performance evaluation of optically preamplified digital pulse position modulation turbulent free-space optical communication systems'. IET Optoelectron., 2012, 6, (1), pp. 66-74

3. Ohtsuki, T.: 'Performance analysis of indoor infrared wireless systems using PPM CDMA'. Electronics and Commun. in Japan (Part I: Communications), 2002, 85, (1), pp. 1-10

4. Leeson, M. S.: 'Pulse position modulation for spectrum-sliced transmission'. IEEE Photonics Technology Letters, , 2004, 16, (4), pp. 1191-1193

5. Miyazawa, T., Sasase, I.: 'BER Performance Analysis of Spectral Phase-Encoded Optical Atmospheric PPM-CDMA Communication Systems'. J. Lightw. Technol., 2007, 25, (10), pp. 2992-3000

6. Garrett, I.: 'Pulse-Position Modulation for Transmission Over Optical Fibers with Direct or Heterodyne Detection'. IEEE Trans. Commun., 1983, 31, (4), pp. 518-527

7. Phillips, A. J., Cryan, R. A., Senior, J. M.: 'Optically preamplified pulse-position modulation for fibre-optic communication systems'. IEE Proc. - Optoelectron., 1996, 143, (2), pp. 153-159

8. Bongtae, K., Byoung-Whi, K.: 'WDM-PON development and deployment as a present optical access solution'. Conf. on Optical Fiber Commun. - incudes post deadline papers, 2009, pp. 1-3

9. Ke, W., Nirmalathas, A., Lim, C., Skafidas, E.: ' 4 x 12.5 Gb/s WDM Optical Wireless Communication System for Indoor Applications'. J. Lightw. Technol., 2011, 29, (13), pp. 1988-1996

10. Aladeloba, A. O., Woolfson, M. S., Phillips, A. J.: 'WDM FSO Network with TurbulenceAccentuated Interchannel Crosstalk'. J. Opt. Commun. Netw., 2013, 5, (6), pp. 641-651

11. Gee-Kung, C., Chowdhury, A., Zhensheng, J., Hung-Chang, C., Ming-Fang, H., Jianjun, Y., Ellinas, G.: 'Key Technologies of WDM-PON for Future Converged Optical Broadband Access Networks [Invited]'. IEEE/OSA J. of Optical Commun. and Networking, 2009, 1, (4), pp. C35-C50

12. Liu, X., Chandrasekhar, S., Wood, T. H., Tkach, R. W., Winzer, P. J., Burrows, E. C., Chraplyvy, A. R.: 'M-ary pulse-position modulation and frequency-shift keying with additional polarization/phase modulation for high-sensitivity optical transmission'. Opt. Express, 2011 2011/12/12, 19, (26), pp. B868-B881

13. Selmy, H., Shalaby, H. M. H., Kawasaki, Z.-I.: 'Proposal and Performance Evaluation of a Hybrid BPSK-Modified MPPM Technique for Optical Fiber Communications Systems'. J. Lightw. Technol., 2013 2013/11/15, 31, (22), pp. 3535-3545

14. Caplan, D. O., Robinson, B. S.: 'WDM Mitigation of Nonlinear Impairments in Low-DutyCycle M-PPM Free-Space Optical Transmitters'. Conf. Optical Fiber commun./National Fiber Optic Engineers Conf., 2008, pp. 1-3

15. Liu, L., Zhang, M., Liu, M., Zhang, X.: 'Experimental demonstration of RSOA-based WDM PON with PPM-encoded downstream signals'. Chin. Opt. Lett., 2012, 10, (7), pp. 070608

16. Tanaka, Y., Nakagawa, M.: 'Optical multi-wavelength PPM for high data rate transmission on indoor channels'. The 8th IEEE Int. Symposium on Personal, Indoor and Mobile Radio Commun., 1997, pp. 979-983 
17. Yanhong, W., Min, Z., Mingtao, L., Lei, L., Ting, S., Xue, C.: '100-km Long-Reach WDM-PON Using High Power Efficient On-Off Keying Codes in Downstream'. Int. Conf. on Control Engineering and Commun. Technol., 2012, pp. 697-699

18. Monroy, I. T., Tangdiongga, E. Crosstalk in WDM communication networks. Norwell, Massachusetts, USA, : Kluwer Academic Publishers; 2002.

19. Ramaswami, R., Sivarajan, K. N., Sasaki, G. H. Optical Networks A Practical Perspective. 3rd ed. Boston: Morgan Kaufmann Publishers; 2010.

20. Ma, R., Zuo, T. J., Sujecki, S., Phillips, A. J.: 'Improved performance evaluation for DCcoupled burst mode reception in the presence of amplified spontaneous emission noise and interchannel crosstalk'. IET Optoelectron., , 2010, 4, (3), pp. 121-132

21. Al-Orainy, A. A., O'Reilly, J. J.: 'Error probability bounds and approximations for the influence of crosstalk on wavelength division multiplexed systems'. IEE Proceedings $J$. Optoelectronics, 1990, 137, (6), pp. 379-384

22. Ke, W., Nirmalathas, A., Lim, C., Skafidas, E.: 'Impact of Crosstalk on Indoor WDM Optical Wireless Communication Systems'. IEEE Photonics Journal, 2012, 4, (2), pp. 375-386

23. Qinglong, Y., Liying, T., Jing, M.: 'Analysis of Crosstalk in Optical Satellite Networks With Wavelength Division Multiplexing Architectures'. J. Lightw. Technol., 2010, 28, (6), pp. 931-938

24. Kedar, D., Arnon, S.: 'Backscattering-induced crosstalk in WDM optical wireless communication'. J. Lightw. Technol., 2005, 23, (6), pp. 2023-2030

25. Lee, Y., Furey, L., Alexander, S., Gallagher, W. F., Salesky, R., Inui, S. Method, system and apparatus for initiating and maintaining synchronization of a pulse position modulation (PPM) decoder with a received PPM signal. Google Patents; 2004

26. Ribeiro, L. F. B., Da Rocha, J. R. F., Pinto, J. L.: 'Performance evaluation of EDFA preamplified receivers taking into account intersymbol interference'. J. Lightw. Technol., 1995, 13, (2), pp. 225-232

27. Personick, S. D.: 'Applications for quantum amplifiers in simple digital optical communication systems'. Bell Syst. Tech. J., 1973, 52, (1), pp. 117-133

28. Phillips, A. J., Cryan, R. A., Senior, J. M.: 'An optically preamplified PPM intersatellite system described by a moment-generating function formulation'. Microwave and Optical Technol. Letters, 1995, 8, (4), pp. 200-204

29. Sibley, M. J. Optical Communications : components and systems. 2nd ed. London: Macmillian Press Ltd; 1995.

30. Maru, K., Mizumoto, T., Uetsuka, H.: 'Demonstration of Flat-Passband Multi/Demultiplexer Using Multi-Input Arrayed Waveguide Grating Combined With Cascaded Mach?Zehnder Interferometers'. J. Lightw. Technol., 2007 2007/08/01, 25, (8), pp. 2187-2197

31. Hirano, A., Miyamoto, Y., Kuwahara, S.: 'Performances of CSRZ-DPSK and RZ-DPSK in 43-Gbit/s/ch DWDM G.652 single-mode-fiber transmission'. Optical Fiber Commun. Conf., 2003, pp. 454-456

32. Yu, C. X., Neilson, D. T.: 'Diffraction-grating-based (de)multiplexer using image plane transformations'. IEEE Journal of Selected Topics in Quantum Electronics,, 2002, 8, (6), pp. 1194-1201

33. Majumdar, A. K.: 'Free-space laser communication performance in the atmospheric channel'. J. Opt. Fiber Commun. Rep., 2005, 2, pp. 345 - 396

34. Shilong, P., Jianping, Y.: 'IR-UWB-Over-Fiber Systems Compatible With WDM-PON Networks'. J. Lightwave Technol., 2011, 29, (20), pp. 3025-3034 
35. Juanjuan, Y., Zheng, Z., Weiwei, H., Anshi, X.: 'Improved Performance of M-ary PPM Free-Space Optical Communication Systems in Atmospheric Turbulence due to Forward Error Correction'. Intl. Conf. on Commun. Technol. 2006, pp. 1-4 